\title{
Neutrinoless $\beta \beta$ decay mediated by the exchange of light and heavy neutrinos: The role of nuclear structure correlations
}

\author{
J. Menéndez \\ Center for Nuclear Study, The University of Tokyo, 113-0033 Tokyo, Japan \\ E-mail: menendez@cns.s.u-tokyo.ac.jp
}

\begin{abstract}
Neutrinoless $\beta \beta$ decay nuclear matrix elements calculated with the shell model and energy-density functional theory typically disagree by more than a factor of two in the standard scenario of light-neutrino exchange. In contrast, for a decay mediated by sterile heavy neutrinos the deviations are reduced to about $50 \%$, an uncertainty similar to the one due to short-range effects. We compare matrix elements in the light- and heavy-neutrino-exchange channels, exploring the radial, momentum transfer and angular momentum-parity matrix element distributions, and considering transitions that involve correlated and uncorrelated nuclear states. We argue that the shorter-range heavy-neutrino exchange is less sensitive to collective nuclear correlations, and that discrepancies in matrix elements are mostly due to the treatment of long-range correlations in many-body calculations. Our analysis supports previous studies suggesting that isoscalar pairing correlations, which affect mostly the longer-range part of the neutrinoless $\beta \beta$ decay operator, are partially responsible for the differences between nuclear matrix elements in the standard light-neutrino-exchange mechanism.
\end{abstract}

Keywords Double-beta decay, nuclear matrix elements, shell model

\section{Introduction}

Searches for nuclear neutrinoless $\beta \beta(0 \nu \beta \beta)$ decay - the process where a nucleus decays into its isobar with two more protons and two fewer neutrons emitting only two electrons - aim to establish that neutrinos are its own antiparticles, a unique property among elementary particles first proposed by Ettore Majorana eight decades ago [1. These experiments are very challenging because the decay they are looking after is indeed very rare, for at least two reasons. First, $\beta \beta$ decay implies a second-order process in the weak interaction, which must be very slow. Second, the neutrinoless mode violates lepton number - two leptons are created - so that there is a further suppression due to the tiny value of the neutrino masses, or in general by any small parameter distinctive of the new physics beyond the Standard Model responsible for the lepton-number-violating decay. While the lepton-number-conserving $\beta \beta$ decay with the additional emission of two antineutrinos has been observed in a dozen nuclei, in some cases with half-lives as short as $10^{19}$ years [2, present lower limits for the neutrinoless mode already indicate half-lives longer than about $10^{26}$ years for ${ }^{136} \mathrm{Xe}[3]$, 
and $10^{25}$ years for ${ }^{76} \mathrm{Ge}\left[4\right.$ and ${ }^{130} \mathrm{Te}[5$. Ongoing searches and future proposals aim to increase the sensitivity of $0 \nu \beta \beta$ decay experiments by several orders of magnitude in the next decade $[6,7,8$.

The $0 \nu \beta \beta$ decay half-life also depends on the associated nuclear matrix element, reflecting that the process occurs within atomic nuclei 9]. Consequently, matrix elements are crucial to anticipate the experimental sensitivity needed to expect a detection signal - within a given model of lepton-number violation - and likewise, to interpret such a decay signal by identifying the underlying mechanism responsible for it. Once the decay channel is known, information can be gained on the corresponding new-physics model. For instance, in the standard scenario that $0 \nu \beta \beta$ decay is mediated by the virtual exchange of the observed light neutrinos, the nuclear matrix elements would allow to identify the arrangement of the neutrino masses - the neutrino hierarchy - and to extract information on the absolute neutrino mass.

Besides the exchange of the light neutrinos we know to exist, other mechanisms involving new physics beyond the Standard Model could trigger the $0 \nu \beta \beta$ decay. These include the existence of lepton and hadron right-handed currents predicted in leftright symmetric models, the exchange of supersymmetric particles, or the emission of Majorons associated with the spontaneous breaking of the baryon-lepton number symmetry [10]. One representative example recently explored in detail in the literature is the exchange of sterile heavy neutrinos via left-handed currents. Sterile neutrinos are predicted in several new-physics models that incorporate the seesaw mechanism to explain the tiny masses of the observed light neutrinos [11. In addition, sterile neutrinos with masses in the $\mathrm{eV}$ range have also been motivated as an explanation to the anomalies seen in a number of neutrino experiments [12, and $\mathrm{keV}$ sterile neutrinos have been proposed as a source of warm dark matter [13. For $0 \nu \beta \beta$ decay mediated by sterile-neutrino exchange, the corresponding nuclear matrix elements can provide access to the sterile-neutrino mass and to the mixing of electron and sterile neutrinos, once the $0 \nu \beta \beta$ decay half-life has been measured.

It is well known that, at present, uncertainties in nuclear matrix element calculations for the light-neutrino-exchange mechanism [14, 15, 16, 17, 18, 19, 20, 21, 22, 23, are so large that its capability to give useful guidance to experiments is in practice limited [9]. This shortcoming would similarly prevent from obtaining reliable information on the neutrino mass and hierarchy in the event of a $0 \nu \beta \beta$ decay signal, assuming the standard scenario for lepton-number violation. There are two main sources of uncertainty in matrix element calculations. First, most of the quoted numbers in the literature ignore a possible "quenching", or renormalization, that would reduce the value of the matrix elements up to a factor two or more. Calculations need such a renormalization factor to correctly reproduce the half-lives of single- $\beta$ decays and $\beta \beta$ decays with the emission of antineutrinos, but its origin is not well understood. Among the most popular explanations are deficiencies in the many-body approaches, and missing two-nucleon (or meson-exchange) terms in the transition operator 9 , 24. The consequences for $0 \nu \beta \beta$ decay matrix elements depend on the origin of the "quenching" 9. The additional contribution of the isotensor axial polarizability to two-nucleon decays was proposed recently [25, 26]. Second, calculations using varied many-body methods severely disagree in their predictions up to a factor two or three depending on the isotope - illustrating the subtle sensitivity of the $0 \nu \beta \beta$ decay operator. In contrast, the same many-body methods usually predict rather consistent nuclear structure observables - such as excitation energy spectra or electromagnetic transitions - across the nuclear chart. 
Much less attention has been devoted to the nuclear matrix element uncertainties associated with decay mechanisms involving new physics beyond the Standard Model. Recently, several calculations have become available for the case of $0 \nu \beta \beta$ decay mediated by the exchange of sterile heavy neutrinos [11, 16, 20, 21, 27, 28, 29, 30, 31, 32, 33. Matrix elements in this channel would be in principle also affected by the uncertainty associated with "quenching". The renormalization specific to this mechanism could be unique because the exchange of virtual heavy neutrinos is characterized by a shorter-range character and larger momentum transfers, on which the "quenching" can depend. This is especially the case if the renormalization is due to meson-exchange currents [34, 35]. In addition, heavy-neutrino-exchange matrix elements obtained with various many-body approaches can be confronted in the same fashion to the routine comparisons performed in the light-neutrino-exchange channel. A combined study of matrix elements in several channels can bring new insights to understand the inconsistencies among present calculations.

In this work we compare shell-model nuclear matrix elements to recently published results obtained with covariant energy-density functional (EDF) theory [32, with focus on both light- and heavy-neutrino-exchange mechanisms. For the heavy-neutrinoexchange channel, the matrix elements depend much less on the many-body method used than in the case of exchange of virtual light neutrinos. In fact, the discrepancies for the heavy-neutrino exchange are comparable to the uncertainty associated with short-range effects. The latter uncertainty is much larger for heavy neutrinos than in the light-neutrino-exchange channel because in the former case larger momentum transfers become relevant, and the short-range part of the operator - at present mostly unexplored - turns out more important [20, 29, 32, 36].

Previous studies have pointed out the relevance of nuclear structure correlations in understanding the deviations between matrix elements obtained with alternative many-body approaches $[9$. The objective of this work is to exploit the distinct nature of the shorter-range heavy-neutrino-exchange and the longer-range light-neutrinoexchange mechanisms to investigate the influence of the leading nuclear structure correlations in each of the two channels. The study of transitions involving simplified uncorrelated states can provide an overall picture of the role played by nuclear correlations [37. In addition, a separable collective Hamiltonian 38, can be used to single out particular correlations [39]. Our analysis aims to identify correlations relevant for the light-neutrino exchange but not so much for the heavy-neutrino case. The contrasting comparison of matrix elements in the two decay channels suggests that the diverse many-body treatment of these correlations can explain part of the disagreement between calculated nuclear matrix elements.

This paragraph completes the introductory Sec. 1. The remaining of the article is structured as follows. In Sec. 2 we introduce the $0 \nu \beta \beta$ decay nuclear matrix elements corresponding to the light- and heavy-neutrino-exchange mechanisms. We compare the results obtained with the shell model and covariant EDF theory for each decay channel in Sec. 3. In addition we investigate the matrix element contributions in terms of the internucleon distance, the momentum transfer, and the quantum numbers of the pair of decaying nucleons. Section 4 discusses the role of nuclear structure correlations. We first focus on matrix elements for decays involving uncorrelated initial and final nuclear states. Subsequently, we use a a separable collective Hamiltonian to explore individual nuclear correlations in terms of their impact on each $0 \nu \beta \beta$ decay mechanism, placing special emphasis on isoscalar pairing correlations. Section 5 summarizes the main findings of the article. 


\section{Light- and heavy-neutrino-exchange mechanisms}

We focus on the $0 \nu \beta \beta$ decay mediated either by the virtual exchange of Standard Model light neutrinos or by sterile heavy-neutrino exchange. Here "heavy" is defined with respect to the typical momentum transfer of the decay, $|\boldsymbol{q}| \sim 100 \mathrm{MeV}$, so that the heavy-neutrino mass satisfies $m_{h} \gg|\boldsymbol{q}|$. References [11, 27, 28, 29, 31] discuss matrix elements for the general case of $0 \nu \beta \beta$ decay mediated by neutrinos of arbitrary mass. Under the assumptions above the half-life of the process can be written as

$$
\left[T_{1 / 2}^{0 \nu}\right]^{-1}=G^{0 \nu}\left(Q_{\beta \beta}, Z\right)\left[\left|M^{0 \nu}\right|^{2} m_{\beta \beta}^{2}+\left|M^{0 N}\right|^{2} \eta_{\beta \beta}^{2}\right],
$$

where $G_{0 \nu}\left(Q_{\beta \beta}, Z\right)$ is a known phase-space factor which depends on the initial and final nuclear energies and the electron mass, $Q_{\beta \beta}=E_{i}-E_{f}-2 m_{e}$, and on the atomic number $Z$ [40]. $M^{0 \nu}$ and $M^{0 N}$ are the nuclear matrix elements of the light- and heavyneutrino-exchange channels, respectively. The parameters $m_{\beta \beta}$ and $\eta_{\beta \beta}$ characterise the lepton-number violation, and are given by

$$
\begin{aligned}
& m_{\beta \beta}=1 / m_{e} \sum_{l=\text { light }} U_{e l} m_{l}, \\
& \eta_{\beta \beta}=m_{N} \sum_{h=\text { heavy }} U_{e h} / m_{h},
\end{aligned}
$$

with $U_{e j}$ the component of the neutrino mixing matrix that connects electron flavour with the light or heavy mass-eigenstate of mass $m_{j}$. The electron and nucleon mass

$m_{N}$ are introduced by convention to make the lepton-number-violation parameters dimensionless.

For both neutrino-exchange channels the matrix elements can be decomposed according to the three separate spin structures of the $0 \nu \beta \beta$ decay operator: $S_{F}=1$, $S_{G T}=\boldsymbol{\sigma}_{a} \cdot \boldsymbol{\sigma}_{b}$ and $S_{T}=3 \boldsymbol{\sigma}_{j} \cdot \hat{\boldsymbol{r}}_{a b} \boldsymbol{\sigma}_{k} \cdot \hat{\boldsymbol{r}}_{a b}-\boldsymbol{\sigma}_{a} \cdot \boldsymbol{\sigma}_{b}$, with $\boldsymbol{\sigma}$ the spin operator and $\hat{\boldsymbol{r}}_{a b}$ the unit vector in the radial direction between the two decaying neutrons. Therefore the full matrix element consists of three parts:

$$
M=M_{G T}-\frac{g_{V}^{2}}{g_{A}^{2}} M_{F}+M_{T},
$$

with dominant Gamow-Teller $\left(M_{G T}\right)$, subleading Fermi $\left(M_{F}\right)$ and smaller tensor $\left(M_{T}\right)$ components. It is relevant to note that calculations without isospin conservation overestimate $M_{F}$ [37. In this work we take vector and axial couplings $g_{V}=1$ and $g_{A}=1.27$ [41]. The three components of the matrix element $M_{X}$ introduced in Eq. (4) can be defined in a general form as a function of the mass of the virtual neutrino exchanged in the decay, $m_{j}$ :

$$
\begin{aligned}
& \lambda_{\beta \beta} M_{X}=\frac{1}{m_{e}} \sum_{j} U_{e j} m_{j} \\
& \quad \times \frac{2 R}{\pi} \int_{0}^{\infty} \boldsymbol{q}^{2} d|\boldsymbol{q}|\left\langle f\left|\sum_{a, b} \frac{j_{X}\left(|\boldsymbol{q}| r_{a b}\right) h_{X}(|\boldsymbol{q}|) S_{X}}{\sqrt{\boldsymbol{q}^{2}+m_{j}^{2}}\left(\sqrt{\boldsymbol{q}^{2}+m_{j}^{2}}+\mu\right)} \tau_{a}^{+} \tau_{b}^{+}\right| i\right\rangle .
\end{aligned}
$$

The lepton-number-violating parameter $\lambda_{\beta \beta}$ is either $m_{\beta \beta}$ or $\eta_{\beta \beta}, j_{G T}=j_{F}=j_{0}$ and $j_{T}=j_{2}$ are spherical Bessel functions, $h_{X}$ are the neutrino potentials [9, 42] given in Appendix A and $R=1.2 A^{1 / 3} \mathrm{fm}$ is introduced by convention to make the matrix elements dimensionless. A sum is performed over all nucleons in the nucleus, with the isospin operator $\tau_{a}^{+}$turning nucleon $a$ into a proton if it is originally a neutron. 
Equation (5) assumes the closure approximation, so that only the initial nuclear state $|i\rangle$ with $Z$ protons and $N$ neutrons and the final nuclear state $|f\rangle$ with $Z+2$ protons and $N-2$ neutrons are needed to evaluate the nuclear matrix element. This approximation entails an additional parameter $\mu$, a representative energy of the states in the intermediate nucleus with $Z+1$ protons and $N-1$ neutrons that enter in the second-order perturbation-theory expression of the matrix element without the closure approximation. Diverse calculations using the non-approximated expression [43, 44, 45] indicate that with reasonable choices of $\mu \sim 10 \mathrm{MeV}$-but with values that slightly depend for each $\beta \beta$ transition - the closure approximation introduces an error of less than $10 \%$ in the light-neutrino-exchange mechanism.

In the limits of light and heavy neutrinos Eq. (5) leads to

$m_{\beta \beta} M_{X}^{0 \nu}=\left(\frac{1}{m_{e}} \sum_{j} U_{e j} m_{j}\right) \frac{2 R}{\pi} \int_{0}^{\infty} \boldsymbol{q}^{2} d|\boldsymbol{q}|\left\langle f\left|\sum_{a, b} \frac{j_{X}\left(|\boldsymbol{q}| r_{a b}\right) h_{X}(|\boldsymbol{q}|) S_{X}}{|\boldsymbol{q}|(|\boldsymbol{q}|+\mu)} \tau_{a}^{+} \tau_{b}^{+}\right| i\right\rangle,(6)$

$\eta_{\beta \beta} M_{X}^{0 N}=\left(m_{N} \sum_{j} \frac{U_{e j}}{m_{j}}\right) \frac{2 R}{\pi} \int_{0}^{\infty} \boldsymbol{q}^{2} d|\boldsymbol{q}|\left\langle f\left|\sum_{a, b} \frac{j_{X}\left(|\boldsymbol{q}| r_{a b}\right) h_{X}(|\boldsymbol{q}|) S_{X}}{m_{N} m_{e}} \tau_{a}^{+} \tau_{b}^{+}\right| i\right\rangle$,

where the parentheses isolate the lepton-number-violation parameters, see Eqs. (2) and (3). The rest of the right-hand side are the expressions for the $0 \nu \beta \beta$ decay nuclear matrix elements.

Usually an additional piece is included to account for short-range correlations missed by the many-body calculation of the initial and final nuclear states. Effectively this amounts to replacing the operators defined by Eqs. (6) and (7) by

$$
\langle f|\hat{O}| i\rangle \rightarrow\left\langle f\left|\hat{O} g\left(r_{a b}\right)\right| i\right\rangle,
$$

with the function $g\left(r_{a b}\right)$ parameterized to compensate for the missing correlations [46]. For the light-neutrino exchange the impact of the short-range correlations is almost negligible, but for the heavy-neutrino exchange the most common parameterizations for $g\left(r_{a b}\right)$ result in a significant matrix element uncertainty. This reflects limitations in constraining the short-range physics in the nuclear many-body calculations.

The heavy-neutrino matrix elements in Eq. (7) involve the four-nucleon diagram, and in addition one- and two-pion contributions included in the $h^{A P}$ and $h^{P P}$ terms in Appendix A. The range of these pion-pole contributions in Eq. (7) is not very different to that of the four-nucleon diagram [32. A fully consistent approach to heavyneutrino exchange is based on chiral effective field theory (EFT) [47, where one- and two-pion-exchange diagrams are predicted to contribute at the same chiral order as the four-nucleon diagram, but are of longer range. Similar chiral EFT predictions in other decay channels involving the exchange of a heavy particle are supported by matrix elements obtained with the quasiparticle random-phase approximation (QRPA) [10]. In the chiral EFT framework the hadronic input associated with the contact and pion-exchange diagrams needs to be determined with lattice QCD [48, 49]. At the moment, however, the relevant information is not known for heavy-neutrino exchange. Nevertheless since the main focus of this work is to compare matrix elements for longand short-range contributions, and to study how nuclear structure correlations operate for both kinds of terms, we restrict to heavy-neutrino matrix elements as defined in Eq. 77). For the same reason, the effects of meson-exchange currents 34 and the isotensor axial polarizability [25, 26] are also neglected. Still we emphasize that a chiral EFT approach including these corrections, like indicated in Ref. [50, should be considered in principle to study heavy-neutrino-exchange nuclear matrix elements. 


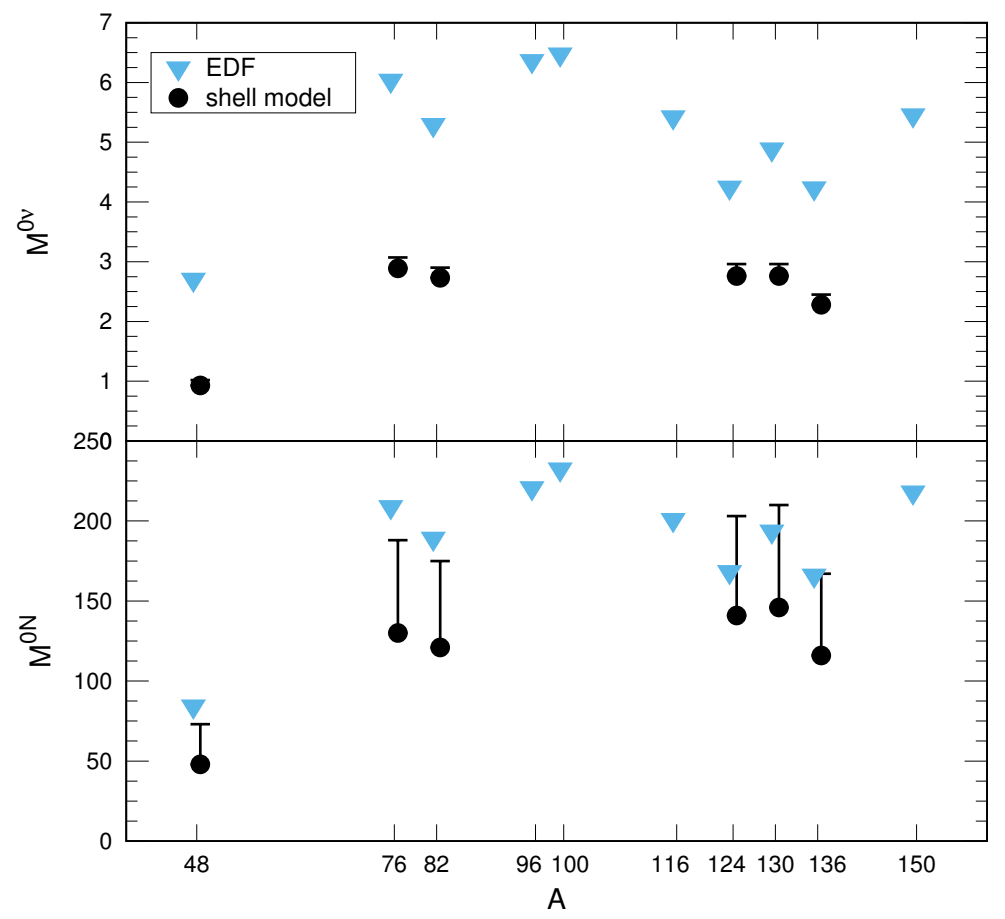

Figure 1. Comparison of nuclear matrix elements obtained with the shell model (black circles) and covariant energy-density functional (EDF) theory [32] (blue triangles) for $\beta \beta$ decay emitters with mass number $A$. Upper panel: Lightneutrino-exchange matrix elements, $M^{0 \nu}$. Lower panel: Heavy-neutrino-exchange matrix elements, $M^{0 N}$. Symbols represent Argonne-type short-range correlations, and shell-model error bars cover results with CD-Bonn-type correlations as well.

\section{Nuclear matrix elements}

Various many-body methods have been employed to study $0 \nu \beta \beta$ decay. In the case of light-neutrino exchange it is well known that different approaches yield matrix elements that vary up to a factor two or more. The shell model typically gives the smallest matrix elements, and EDF theory usually the largest ones [9]. The upper panel of Fig. 1 illustrates this comparing shell-model matrix elements with the covariant EDF theory ones of Ref. 32. The shell-model values are shown in Table 1 .

The shell-model calculations use the same configuration spaces and interactions as Refs. 11, 14, [51]: For ${ }^{48} \mathrm{Ca}$ the neutron and proton single-particle harmonic oscillator orbitals $0 f_{7 / 2}, 1 p_{3 / 2}, 1 p_{1 / 2}$ and $0 f_{5 / 2}$ - the $p f$ shell- with the KB3G interaction [52]; for ${ }^{76} \mathrm{Ge}$ and ${ }^{82} \mathrm{Se}$, the $1 p_{3 / 2}, 1 p_{1 / 2}, 0 f_{5 / 2}$ and $0 g_{9 / 2}$ orbitals with the GCN2850 interaction [14; and for ${ }^{124} \mathrm{Sn},{ }^{130} \mathrm{Te}$ and ${ }^{136} \mathrm{Xe}$, the $0 g_{7 / 2}, 1 d_{5 / 2}, 1 d_{3 / 2}$, $2 s_{1 / 2}$ and $0 h_{11 / 2}$ orbitals with the GCN5082 interaction [14. These shell-model interactions are based on G-matrix interactions [53] derived from realistic nucleonnucleon potentials, with phenomenological modifications, mostly in the monopole part, to accommodate better agreement with spectroscopic data. In particular, the GCN2850 and GCN5082 interactions [54 describe well spectra and electromagnetic properties 55, 56, 57, 58. All shell-model calculations have been performed with the $J$-coupled code NATHAN [59], especially suited for the computation of $0^{+}$states. 
In order to understand the origin of the inconsistency between matrix element calculations, it is useful to confront the results for the heavy-neutrino-exchange channel as well, as in the lower panel of Fig. 1. A comparison of the upper and lower panels in Fig. 1 highlights that the relative variance between matrix elements is much smaller when virtual heavy neutrinos are exchanged than in the standard scenario of light-neutrino exchange. Actually we expect the disagreement to be even milder, because the covariant EDF theory calculations do not preserve isospin as a good quantum number, and the corresponding matrix elements - which are larger than the shell model ones - can be estimated to be about 10\% too large because of this approximation [37. Moreover, for heavy neutrinos the discrepancy is comparable to the uncertainty due to the treatment of short-range correlations - CD-Bonn- and Argonne-type parameterizations [46] are considered - represented by the error bars in the shell-model results in Fig. 1. What causes the contrasting comparison of matrix elements in the two channels?

To answer this question, it is useful to have in mind the distinct nature of the two decay mechanisms. For that purpose, Fig. 2 compares the corresponding normalized radial and momentum transfer matrix element distributions, $C\left(r_{a b}\right)$ and $C(|\boldsymbol{q}|)$, for the representative case of the ${ }^{136} \mathrm{Xe}$ decay. The distributions - without normalizationsatisfy $M=\int C\left(r_{a b}\right) d r_{a b}$ and $M=\int C(|\boldsymbol{q}|) d|\boldsymbol{q}|$. The left panel of Fig. 2 shows that even though the light-neutrino exchange is of relatively short-range, with typical distances between the decaying neutrons of the order of a couple of fm's, the heavyneutrino exchange only allows shorter-range contributions up to $1 \mathrm{fm}$. Alternatively, the right panel of Fig. 2 shows that the heavy-neutrino exchange probes momentum transfers considerably larger than the ones below $|\boldsymbol{q}| \approx 200$ which are the most relevant for light-neutrino exchange. Consequently the influence of short-range correlations in the light-neutrino-exchange channel is minor. In contrast, for momentum transfers $|\boldsymbol{q}| \approx 500 \mathrm{MeV}$, only relevant with heavy neutrinos, short-range correlations play a much more important role. Here the two prescriptions of short-range correlations reduce the value of the matrix elements by significantly different amounts, as shown by the error bars in the lower panel of Fig 1 . The comparison of normalized distributions between the two decay channels agrees well with the findings of QRPA, EDF theory and other many-body approaches [20, 29, 32, 36.

We can also analyse the matrix elements in terms of the contributions from the decaying pair of neutrons - or the created pair of protons - coupled to a given angular momentum and parity, $M\left(J^{\pi}\right)$, with $M=\sum_{J^{\pi}} M\left(J^{\pi}\right)$. Figure 3, taking ${ }^{136} \mathrm{Xe}$ as a representative example, shows that the decomposition is quite diverse in the two channels. For the exchange of light neutrinos the total matrix element results from a significant cancellation of the dominant $0^{+}$-pair term by all other contributions,

Table 1. Shell-model nuclear matrix elements for the exchange of virtual light and heavy neutrinos, $M^{0 \nu}$ and $M^{0 N}$, respectively. Left (right) results obtained with Argonne-type (CD-Bonn-type) short-range correlations.

\begin{tabular}{lcccccc}
\hline & ${ }^{48} \mathrm{Ca}$ & ${ }^{76} \mathrm{Ge}$ & ${ }^{82} \mathrm{Se}$ & ${ }^{124} \mathrm{Sn}$ & ${ }^{130} \mathrm{Te}$ & ${ }^{136} \mathrm{Xe}$ \\
\hline$M^{0 \nu}$ & $0.93 / 1.02$ & $2.89 / 3.07$ & $2.73 / 2.90$ & $2.76 / 2.96$ & $2.76 / 2.96$ & $2.28 / 2.45$ \\
$M^{0 N}$ & $48 / 73$ & $130 / 188$ & $121 / 175$ & $141 / 203$ & $146 / 210$ & $116 / 167$ \\
\hline
\end{tabular}


Neutrinoless $\beta \beta$ decay mediated by light and heavy neutrinos: Role of correlations

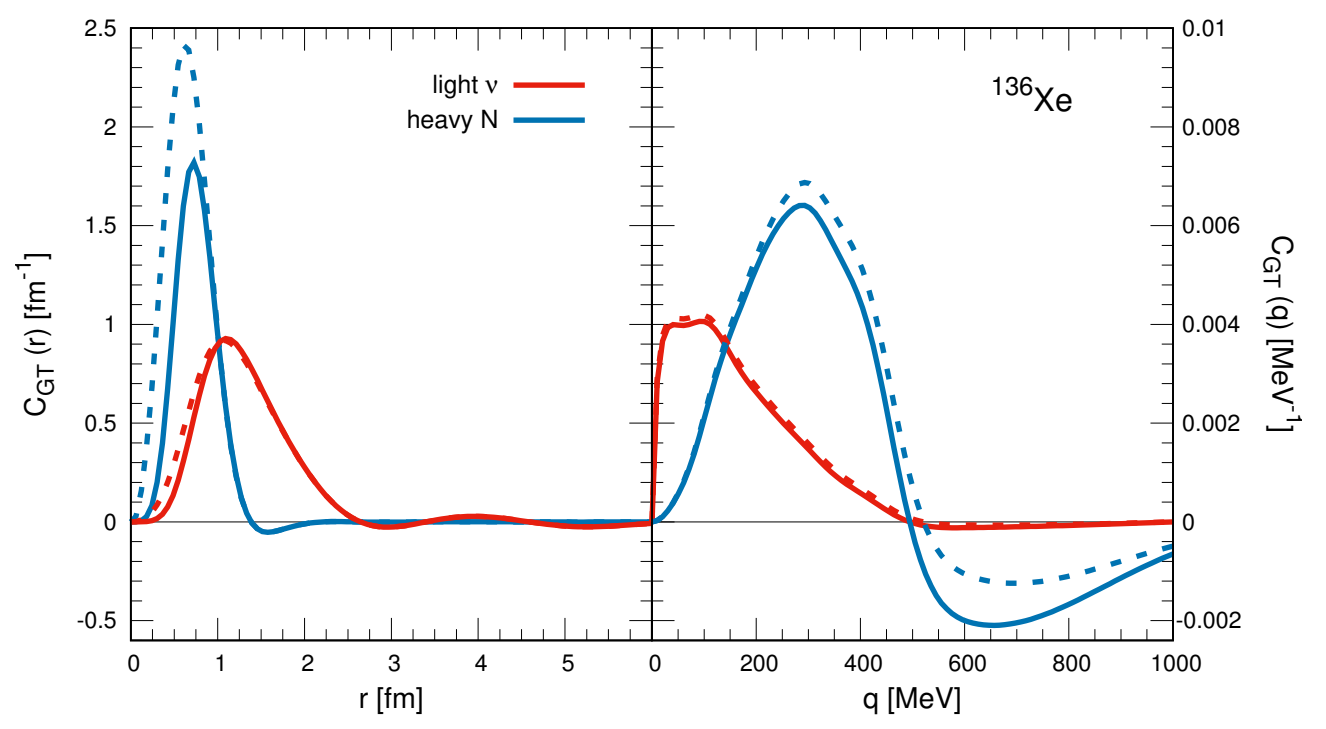

Figure 2. Distribution of the Gamow-Teller matrix element of ${ }^{136}$ Xe. Left panel: Radial distribution. Right panel: Momentum transfer distribution. Red (blue) lines show results for the mechanism that exchanges virtual light (heavy) neutrinos. Solid (dashed) lines represent Argonne-type (CD-Bonn-type) shortrange correlations. Distributions normalized with respect to Argonne-type results.

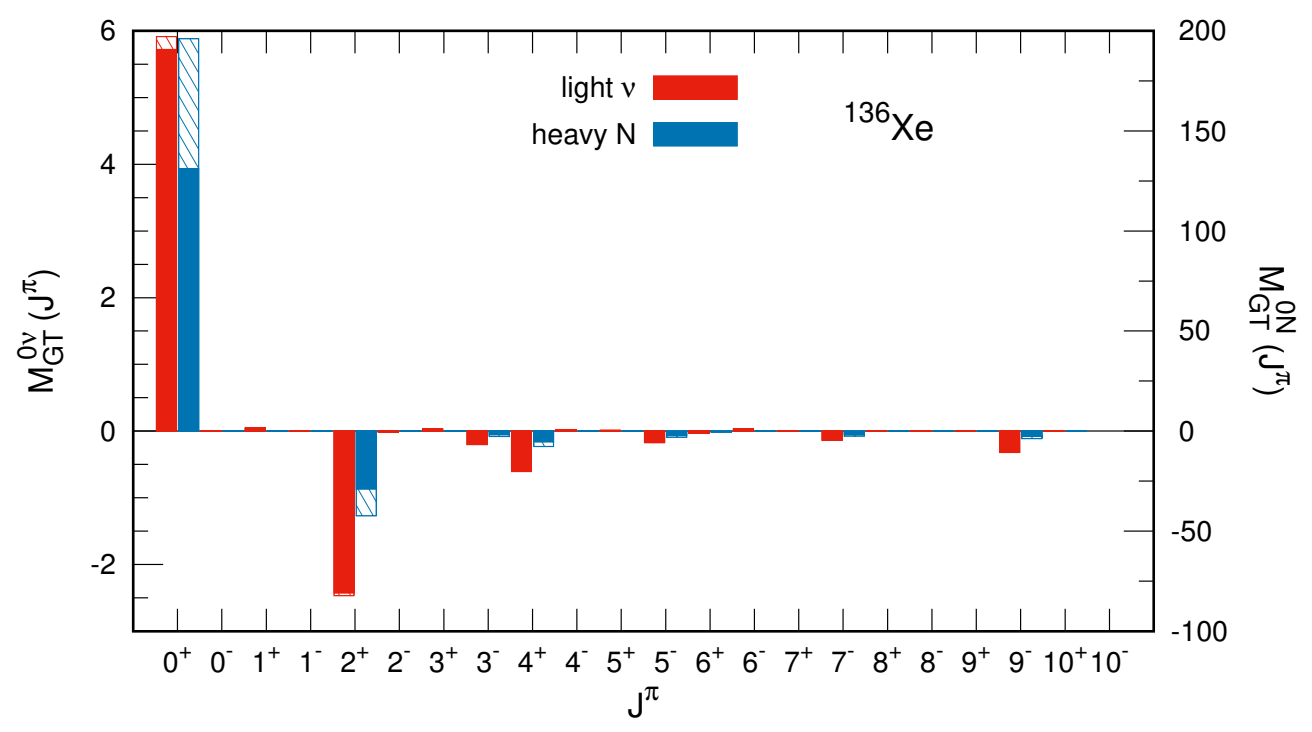

Figure 3. Contributions to the Gamow-Teller matrix element of ${ }^{136} \mathrm{Xe}$ from pairs of decaying neutrons with given angular momentum and parity, $J^{\pi}$. Red (blue) bars show the results for the exchange of virtual light (heavy) neutrinos, $M_{G T}^{0 \nu}\left(J^{\pi}\right)$, left scale $\left[M_{G T}^{0 N}\left(J^{\pi}\right)\right.$, right scale]. Solid (dashed) bars correspond to Argonne-type (CD-Bonn-type) short-range correlations. 
especially those from $2^{+}$pairs. The final result is about $35 \%$ of the $0^{+}$-pair matrix element. In comparison, for the heavy-neutrino exchange the final result is about $70 \%$ of the $0^{+}$-pair matrix element. This term is only modified significantly by $2^{+}$-pair matrix elements, as the contributions of pairs coupled to higher angular momentum are very much suppressed. These findings are in agreement with Refs. 21, 30, 45]. The dominance of the $0^{+}$-pair matrix element is related to the short-range character of the heavy-neutrino exchange because for higher angular momenta shorter-range contributions are much less dominant than for $0^{+}$pairs [60.

The smaller cancellations in the heavy-neutrino exchange matrix elements suggest that this channel is not as subtle as the light-neutrino exchange, where the competition between matrix element contributions needs to be described carefully. This is consistent with the main finding of Fig. 1. Matrix elements for the exchange of heavy neutrinos are similar for the shell model and covariant EDF theory. Figure 2 suggests that most of the matrix element cancellations occur at the level of longerrange correlations involving nucleons separated by a couple of fm's.

It is useful to explain the matrix element cancellations invoking an approximate $S U(4)$ symmetry [39, because when the symmetry is exact in the transition operator and the initial and final nuclear states, the matrix elements vanish. In this picture, the relatively minor cancellation in heavy-neutrino exchange shown in Fig. 3 occurs because the very short-range radial part of the $0 \nu \beta \beta$ decay operator, $\sim \delta\left(r_{a b}\right)$, breaks the $S U(4)$ symmetry more efficiently than the longer-range $\sim 1 / r_{a b}$ radial part corresponding to the light-neutrino exchange. The $S U(4)$ symmetry can only be fully preserved for an operator without $r_{a b}$ dependence.

\section{Role of nuclear structure correlations}

\subsection{Nuclear matrix elements for uncorrelated nuclear states}

Given the differences between matrix elements corresponding to light- and heavyneutrino exchange, we can expect that nuclear structure correlations play alternative roles in each channel. Figure 4 confronts shell-model and covariant EDF theory matrix elements for calculations restricted to uncorrelated states, for both mechanisms: Lightneutrino exchange in the upper panel and heavy-neutrino exchange in the lower panel. In the shell model the initial and final nuclear states are limited to configurations formed by neutron-neutron and proton-proton $0^{+}$pairs - seniority-zero states - while for the covariant EDF theory only spherical states are considered.

First, we note that both Figs. 1 and 4 show a similar matrix element distribution with respect to the mass number in the upper and lower panels, this is, in the two decay channels. This suggests a proportionality between light- and heavy-neutrinoexchange matrix elements obtained with the same many-body approach, a dependence first observed in the QRPA [61]. The non-relativistic EDF theory work Ref. 62] found a similar proportionality when comparing matrix elements for light-neutrino exchange with those corresponding to the same operator with the radial part replaced by the identity, a relation studied in more detail in the context of the correlation between $0 \nu \beta \beta$ decay and double Gamow-Teller transitions [63. These relations suggest that the relative value of the matrix elements depends mostly on the spin-isospin structure and correlations of the nuclear states, and only moderately on the radial part of the operator. In every channel proposed for $0 \nu \beta \beta$ decay -including those corresponding to left-right symmetric and supersymmetric models, and Majoron emission- the 
Neutrinoless $\beta \beta$ decay mediated by light and heavy neutrinos: Role of correlations 10

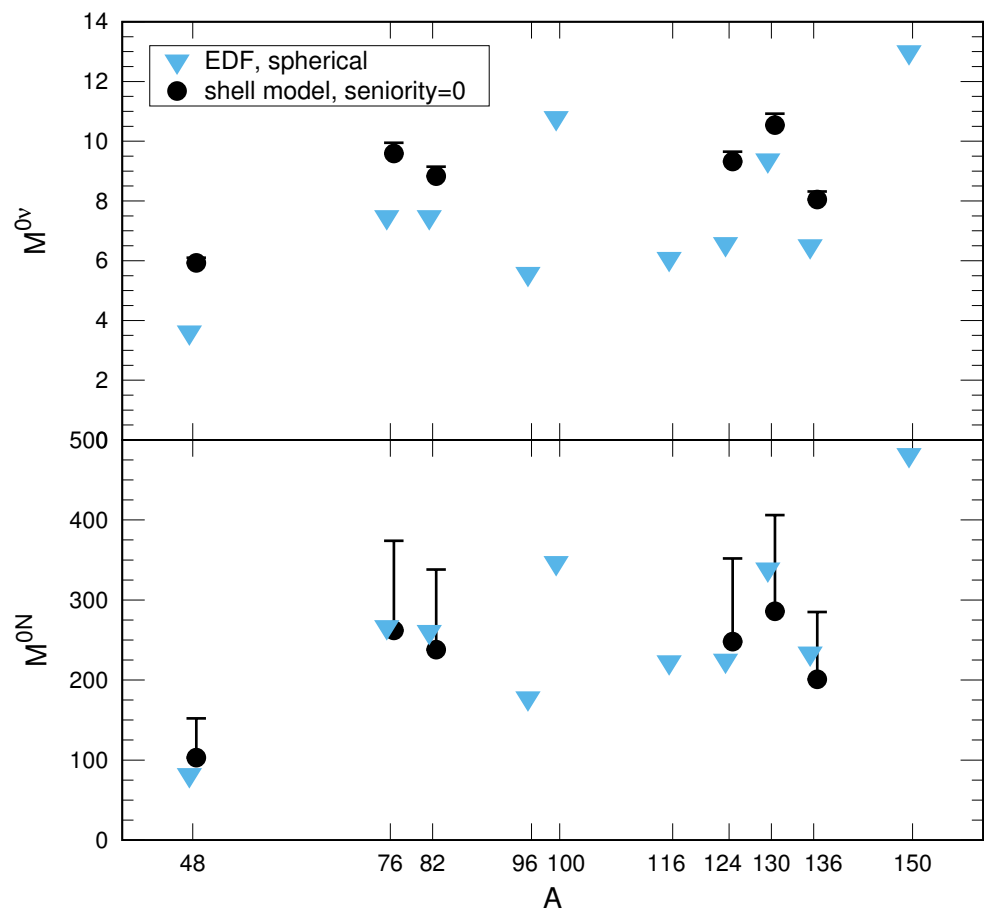

Figure 4. Comparison of nuclear matrix elements obtained with the shell model restricted to seniority-zero states (black circles), and covariant energy-density functional (EDF) theory limited to spherical configurations [32] (blue triangles), for $\beta \beta$ emitters with mass number $A$. Upper panel: Light-neutrino-exchange matrix elements, $M^{0 \nu}$. Lower panel: Heavy-neutrino-exchange matrix elements, $M^{0 N}$. Symbols correspond to Argonne-type short-range correlations, and shellmodel error bars cover results with CD-Bonn-type correlations as well.

dominant spin-isospin structure is a Gamow-Teller-like operator. Therefore, following Refs. 61, 64] we anticipate that it will be challenging to identify the underlying decay mechanism based on a combination of half-life measurements and matrix element calculations in several isotopes, as proposed in Refs. 65, 66]. Reference [50] has very recently obtained a similar conclusion. In principle small deviations from an exact proportionality would allow one to distinguish the decay channel [67, but a reliable identification demands more accurate matrix elements than what they are now.

More importantly, it is apparent in the two panels in Fig. 4 that for uncorrelated states the agreement between shell-model and covariant EDF theory matrix elements is very good. Differences are less than $30 \%$ in the light-neutrino-exchange channel shown in the upper panel - and only about $10 \%$ for the exchange of heavy neutrinos, as the lower panel shows. Moreover the uncorrelated shell model matrix elements can be larger than the EDF theory ones, in contrast to Fig. 1. This highlights that the nuclear correlations in the the shell model impact the nuclear matrix elements much more than those included by EDF theory. The slightly larger shell model values may be related to a stronger pairing in the shell model interaction with respect to the pairing strength of the EDF 37. Note that isospin is not a good quantum number for the uncorrelated states in any of the two many-body methods, and this approximation is expected to impact similarly both approaches. 
Neutrinoless $\beta \beta$ decay mediated by light and heavy neutrinos: Role of correlations 11

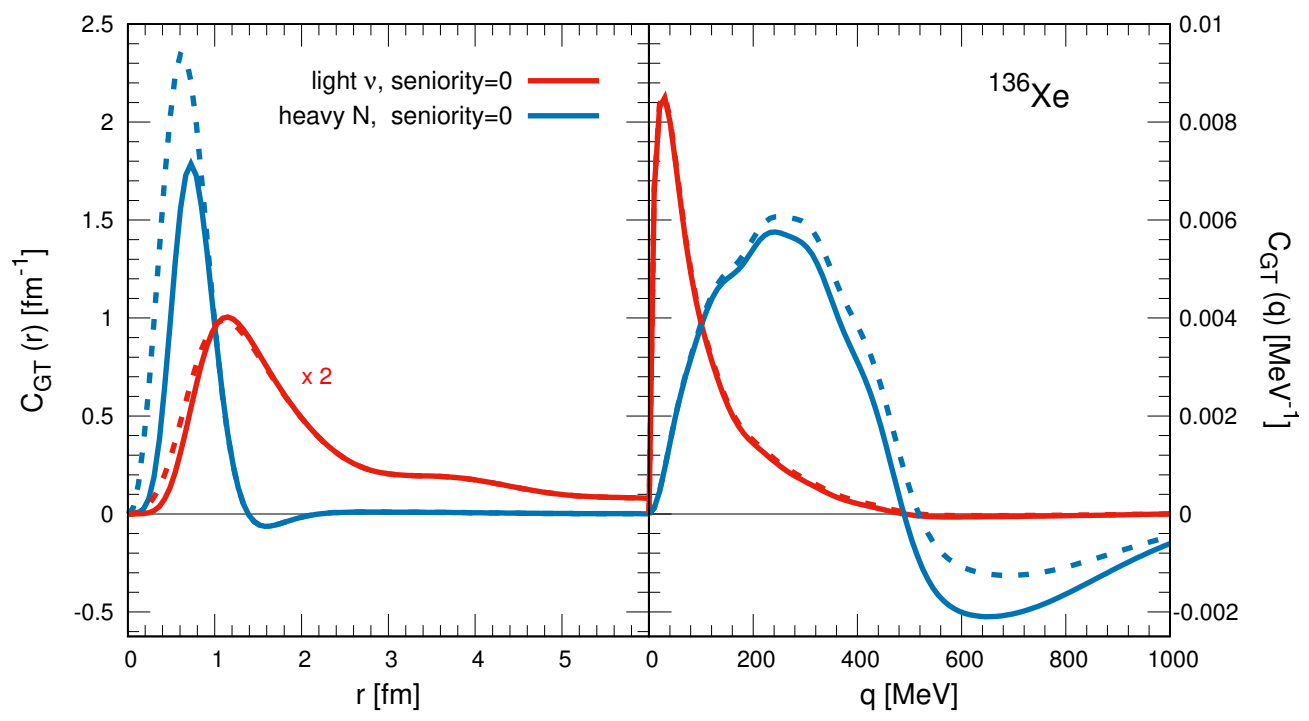

Figure 5. Distribution of the Gamow-Teller matrix element of ${ }^{136} \mathrm{Xe}$ restricted to seniority-zero states. Left panel: Radial distribution, normalized to total value 2 for light neutrinos. Right panel: Momentum transfer distribution. Red (blue) lines show results for the mechanism that exchanges light (heavy) neutrinos. Solid (dashed) lines represent Argonne-type (CD-Bonn-type) short-range correlations. The distributions are normalized with respect to Argonne-type results.

Reference [37] found a similar matrix element consistency for the decay of uncorrelated calcium, titanium and chromium isotopes comparing the shell model and non-relativistic EDF theory. Figure 4 expands this agreement and suggests that it could be extended along the nuclear chart At this level of uncorrelated states the results only depend on single-particle degrees of freedom and like-particle - isovectorpairing correlations. The single-particle structure of non-relativistic EDF theory in comparison to the shell model has been explored in Refs. 69, 70, finding reasonably similar orbital occupancies obtained with both many-body approaches. Likewise, isovector pairing is also fairly consistent in typical shell-model interactions and the Gogny EDF [59. Additional like-pairing correlations can be incorporated by extending the shell-model configuration space 22, 71] or adding isovector pairing fluctuations to the EDF theory calculation [15]. For both methods adding these correlations results in a comparable enhancement of the matrix element values of the order of $30 \%$ in the $0 \nu \beta \beta$ decays studied. All these similarities suggest that the discrepancy between shell-model and EDF theory matrix elements in the standard light-neutrinoexchange scenario is related to the unequal treatment of additional nuclear structure correlations.

The left and right panels of Fig. 5 show the normalized radial and momentum transfer matrix element distributions $C\left(r_{a b}\right)$ and $C(|\boldsymbol{q}|)$, respectively, for uncorrelated seniority-zero nuclear states. The Gamow-Teller ${ }^{136} \mathrm{Xe}$ matrix element is taken as a representative example. In the heavy-neutrino-exchange channel the distributions are very similar to those for fully-correlated states, see the blue lines in the corresponding

$\ddagger$ An exception may be heavy systems like ${ }^{150} \mathrm{Nd}$ where even calculations with spherical states using non-relativistic 68] and covariant [32] EDF theory differ. 
panels in Figs. 5 and 2. In contrast, for the exchange of light neutrinos, a comparison of the red lines in Figs. 5 and 2 shows that when seniority-zero states are involved longer internucleon distances and smaller momentum transfers become relevant. This suggests that additional nuclear correlations effectively suppress the longer-range contributions to the matrix element. Therefore, short-range correlations beyond the seniority-zero level seem to be relatively under control in what respects $0 \nu \beta \beta$ decay - shell-model and EDF theory matrix elements in the heavy-neutrino channel agree reasonably well, see the lower panel in Fig. 11. On the contrary, the discrepancies in the standard light-neutrino-exchange scenario - highlighted by the upper panel in Fig. 1 - seem to be caused mainly by nuclear structure correlations involving nucleons separated by several fm's. Which are these correlations?

\subsection{Isoscalar pairing correlations}

Nuclear interactions usually comprise various collective correlations encoded in the interaction two- and three-nucleon matrix elements. However, the individual correlations are usually not easy to disentangle. An exception is the separable collective Hamiltonian $H_{\text {coll }}$ obtained in Ref. [38. This Hamiltonian is valid in the $p f$ shell configuration space and applicable to nuclei with mass number between $A=40$ and $A \approx 60$. It was constructed to reproduce the most important physics of the shellmodel interaction KB3G. In fact, for the decay of calcium, titanium and chromium isotopes $H_{\text {coll }}$ gives nuclear matrix elements very similar to those of KB3G in the standard light-neutrino-exchange channel [39].

The separable collective Hamiltonian $H_{\text {coll }}$ includes the single-particle behaviour from the monopole part of the KB3G interaction, and the dominant collective correlations:

$$
\begin{aligned}
& H_{\text {coll }}=H_{M}+g^{T=1} \sum_{n=-1}^{1} S_{n}^{\dagger} S_{n}+g^{T=0} \sum_{m=-1}^{1} P_{m}^{\dagger} P_{m} \\
& +g_{p h} \sum_{m, n=-1}^{1}: \mathcal{F}_{m n}^{\dagger} \mathcal{F}_{m n}:+\chi \sum_{\mu=-2}^{2}: Q_{\mu}^{\dagger} Q_{\mu}: .
\end{aligned}
$$

Colons indicate normal ordering, $H_{M}$ describes the single-particle part and

$$
\begin{aligned}
S_{n}^{\dagger} & =\frac{1}{\sqrt{2}} \sum_{\alpha} \sqrt{2 l_{\alpha}+1}\left(a_{\alpha}^{\dagger} a_{\alpha}^{\dagger}\right)_{0,0, n}^{0,0,1}, \\
P_{m}^{\dagger} & =\frac{1}{\sqrt{2}} \sum_{\alpha} \sqrt{2 l_{\alpha}+1}\left(a_{\alpha}^{\dagger} a_{\alpha}^{\dagger}\right)_{0, m, 0}^{0,1,0}, \\
\mathcal{F}_{m n} & =2 \sum_{\alpha} \sqrt{2 l_{\alpha}+1}\left(a_{\alpha}^{\dagger} \tilde{a}_{\alpha}\right)_{0, m, n}^{0,1,1}, \\
Q_{\mu} & =\frac{1}{\sqrt{5}} \sum_{\alpha, \beta}\left\langle n_{\alpha} l_{\alpha}\left\|r^{2} Y_{2} / b^{2}\right\| n_{\beta} l_{\beta}\right\rangle\left(a_{\alpha}^{\dagger} \tilde{a}_{\beta}\right)_{\mu, 0,0}^{2,0,0},
\end{aligned}
$$

where $a_{\alpha}^{\dagger}$ creates a nucleon in a single-particle orbital with $n_{\alpha}$ principal quantum number and $l_{\alpha}$ orbital angular momentum, $\tilde{a}_{a}$ destroys a nucleon in the time-reversed orbital, $Y$ is a spherical harmonic and $b$ the harmonic-oscillator parameter. Values for the strengths of the isovector pairing, isoscalar pairing, spin-isospin and quadrupole terms $g^{T=1}, g^{T=0}, g_{p h}$ and $\chi$, respectively, are given in Ref. [39]. In that reference $H_{\text {coll }}$ was used to isolate the effect of the individual correlations in the standard $0 \nu \beta \beta$ 


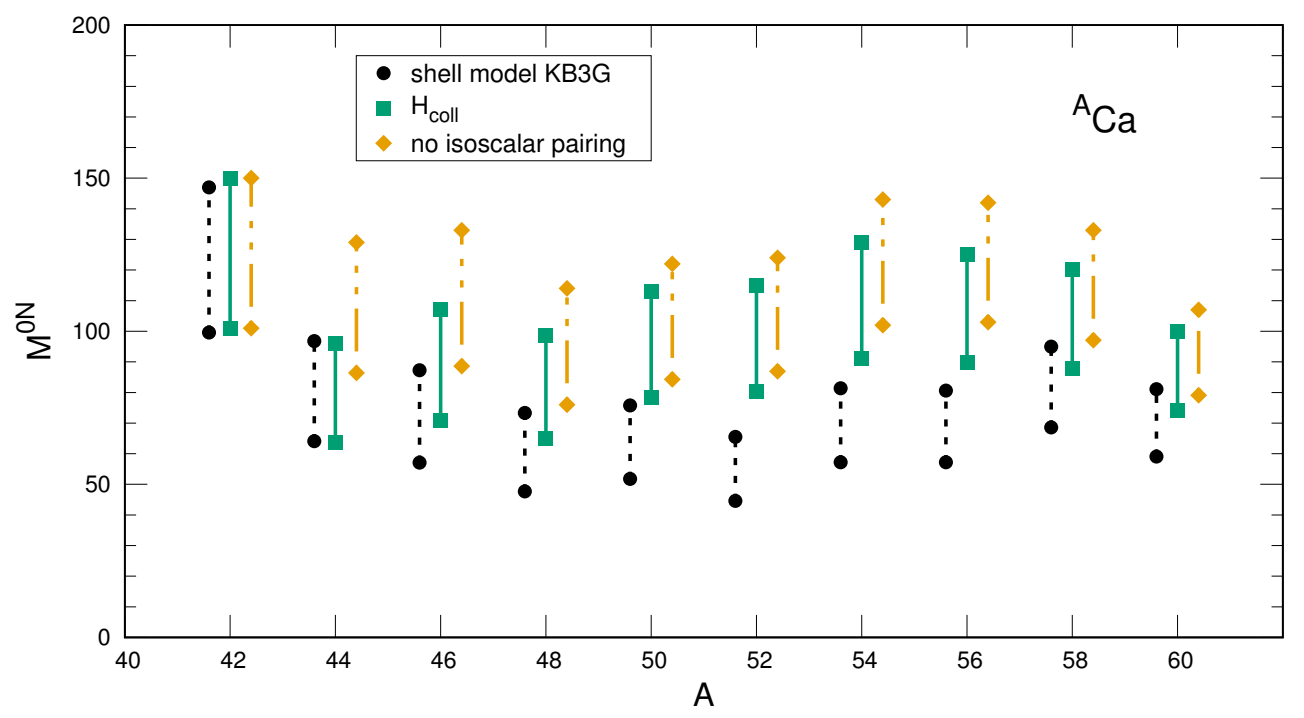

Figure 6. Matrix elements for calcium ${ }^{A} \mathrm{Ca}$ isotopes in the heavy-neutrinoexchange channel, $M^{0 N}$, obtained with the shell-model interaction KB3G (black circles), the collective Hamiltonian $H_{\text {coll }}$ (green squares), and $H_{\text {coll }}$ without the isoscalar pairing term (orange diamonds). Error bars cover results corresponding to Argonne- (lower end) and CD-Bonn-type (upper end) short-range correlations.

decay channel. What is the influence of each of these collective correlations when the transition is mediated by heavy neutrinos?

Isoscalar pairing correlations were shown long time ago to be particularly relevant for $\beta \beta$ decay in the QRPA framework [72, 73, an extreme confirmed in improved recent studies [74, and also with the shell model using $H_{\text {coll }}$ [39]. If isoscalar pairing correlations are not taken into account, the light-neutrino-exchange matrix elements tend to be overestimated. Here we investigate the impact of isoscalar pairing in the heavy-neutrino-exchange mechanism. According to the better agreement between shell model and covariant EDF theory in this channel, if the discrepancies related to the light-neutrino exchange are due to isoscalar pairing, the importance of these correlations would be reduced for the exchange of heavy neutrinos.

Following a similar strategy to Ref. 39] we isolate the effect of the various terms in $H_{\text {coll }}$ by including them or not in the shell model calculation. Figure 6 shows that isoscalar pairing correlations indeed play a relatively small role for the heavyneutrino-exchange mechanism. Matrix elements for the decay of calcium isotopes are enhanced by about $10 \%-30 \%$ when these correlations are omitted, a correction much less important than the uncertainty due to short-range correlations represented by the error bars in Fig. 6. The enhancement is even smaller than the difference between matrix elements corresponding to $H_{\text {coll }}$ and to the KB3G interaction. The impact of isoscalar pairing correlations in the matrix elements associated with the decay of titanium and chromium isotopes is very similar. This minor influence contrasts with the variations observed in Ref. [39] for the $0 \nu \beta \beta$ decay of the same nuclei in the standard light-neutrino-exchange channel. In that reference, the matrix elements obtained without isoscalar pairing were found to be a factor of two or three larger than those corresponding to $H_{\text {coll }}$ or KB3G. 
Neutrinoless $\beta \beta$ decay mediated by light and heavy neutrinos: Role of correlations 14

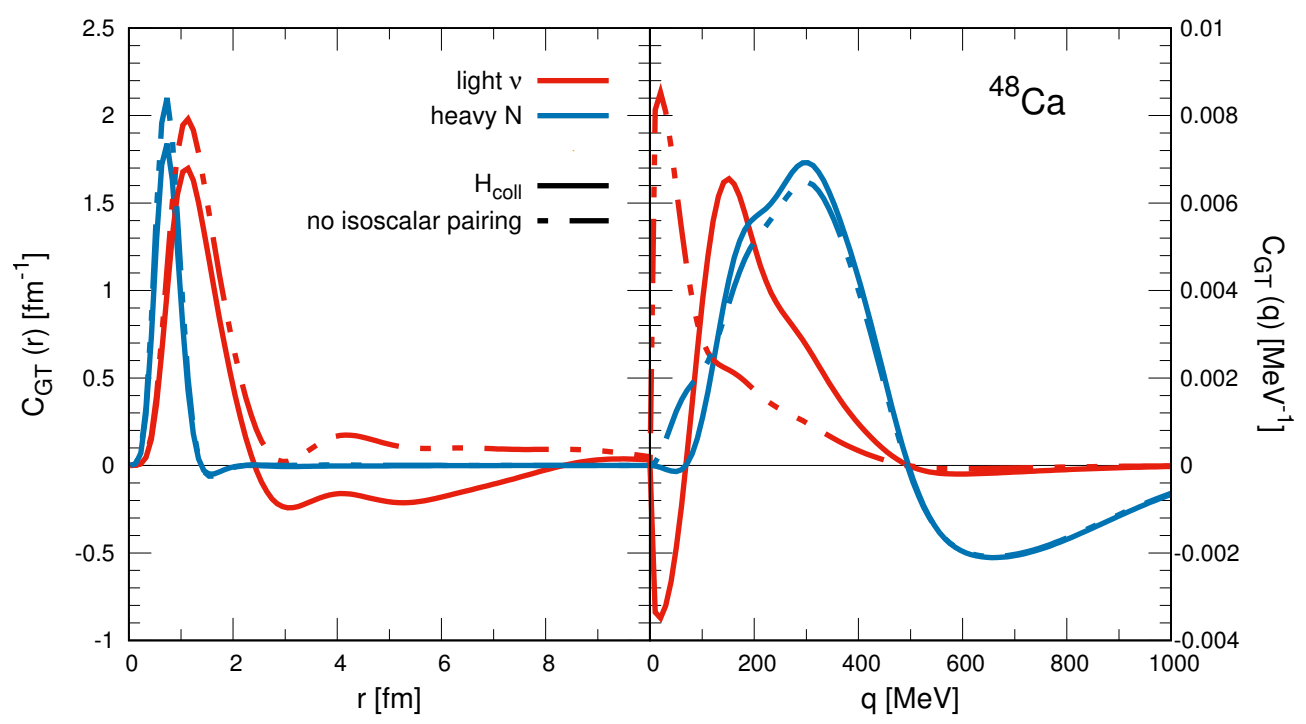

Figure 7. Distribution of the Gamow-Teller matrix element of ${ }^{48} \mathrm{Ca}$. Left panel: Radial distribution. Right panel: Momentum transfer distribution. Red (blue) lines represent the mechanism that exchanges virtual light (heavy) neutrinos. Solid (dashed) lines show results for the collective Hamiltonian $H_{\text {coll }}$ including (excluding) the isoscalar pairing term. All results use Argonne-type short-range correlations. The distributions are normalized with respect to $H_{\text {coll }}$ results.

The sensitivity of the heavy-neutrino-exchange matrix elements to the remaining terms in $H_{\text {coll }}$ - the Landau-Migdal-style spin-isospin and quadrupole correlationsturns out also very small in all the decays of $p f$-shell nuclei studied. This is in good agreement with the behaviour found in Ref. [39] in the standard scenario involving light neutrinos. Furthermore, increasing the isovector pairing strength $g^{T=1}$ by $20 \%$ results in a heavy-neutrino matrix element enhancement of a similar amount, which is comparable to the increase found when light neutrinos are exchanged. This hints that the role of like-particle pairing is similar in both channels. In conclusion, our analysis based on the dominant collective nuclear correlations considered in $H_{\text {coll }}$ suggests that one of the main differences between the light- and heavy-neutrino-exchange mechanisms resides in the importance of isoscalar pairing correlations.

Note that, in addition, quadrupole correlations, which are of long range, could also cause differences between the two channels. Quadrupole correlations have been shown to be relevant in several $0 \nu \beta \beta$ decay works, including shell model and EDF theory studies [17, 75, 76, 77, 78. In general, calculations find reduced matrix elements when the initial and final nuclear states exhibit different deformations, a prediction which seems to be in conflict with measurements of $\beta \beta$ decay with antineutrino emission [79. Unfortunately, quadrupole correlations play a small role in the moderately-deformed $p f$-shell nuclei, and they are relatively unimportant for the decays shown in Fig. 66 39. Considering decays involving more deformed systems is necessary to understand better the influence of quadrupole correlations.

Figure 7 shows the radial and momentum transfer matrix element distributions, $C\left(r_{a b}\right)$ and $C(|\boldsymbol{q}|)$ in the two decay channels. The only $\beta \beta$ emitter in the $p f$-shell, ${ }^{48} \mathrm{Ca}$, is taken as an example. We compare calculations performed using $H_{\text {coll }}$ with 
Neutrinoless $\beta \beta$ decay mediated by light and heavy neutrinos: Role of correlations 15

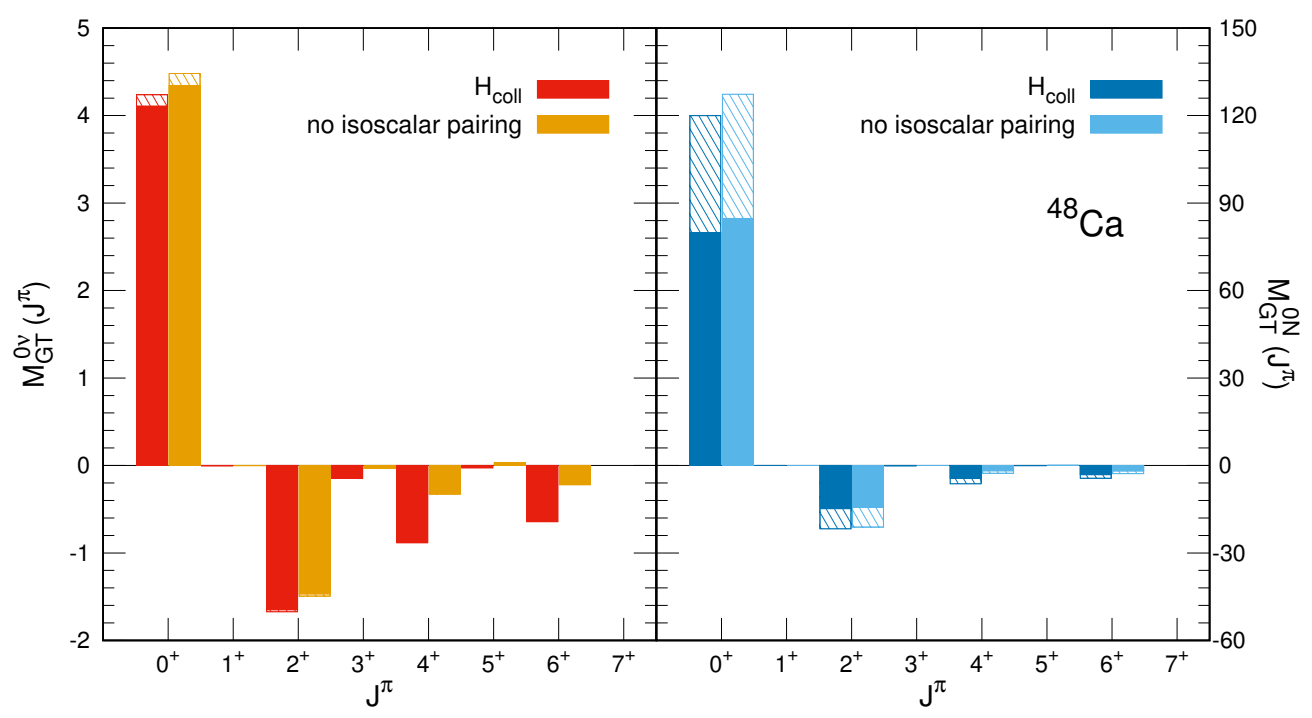

Figure 8. Contributions to the Gamow-Teller matrix element of ${ }^{48} \mathrm{Ca}$ from pairs of decaying neutrons with given angular momentum and parity, $J^{\pi}$. Left panel: Light-neutrino-exchange matrix elements, $M_{G T}^{0 \nu}\left(J^{\pi}\right)$. Right panel: Heavyneutrino-exchange matrix elements, $M_{G T}^{0 N}\left(J^{\pi}\right)$. Red and blue (orange and light blue) bars show results for $H_{\text {coll }}$ ( $H_{\text {coll }}$ without isoscalar pairing). Solid (dashed) bars correspond to Argonne-type (CD-Bonn-type) short-range correlations.

and without the isoscalar pairing term. The left panel of Fig. 7 shows that for the exchange of light neutrinos the impact of isoscalar pairing is significant at longer internucleon distances, inducing matrix element cancellations beyond $3 \mathrm{fm}$ 's or so. When isoscalar pairing is included, the two decaying neutrons can belong to different neutron-neutron and neutron-protons pairs, making this interaction relevant at long distances [80. In comparison, there is only a mild reduction of the short-range matrix element distribution when heavy neutrinos are exchanged. Likewise, the right panel of Fig. 7 shows that the variations in the momentum transfer distribution in the standard channel are more marked for small momentum transfers, where isoscalar pairing correlations reduce the value of the matrix element. For heavy-neutrino exchange the momentum transfer distribution is very much alike for the two Hamiltonians.

In conclusion, Fig. 7 highlights that most of the contributions of isoscalar pairing affect the long-range part of the $0 \nu \beta \beta$ decay operator, which is only important when light neutrinos are exchanged. This implies a more pronounced role of isoscalar pairing correlations in the standard light-neutrino-exchange scenario. As originally motivated in Fig. 1. this is the channel where the variance between matrix elements is more marked. In consequence, our analysis suggests that isoscalar pairing correlations can be responsible for a sizeable part of the well-known disagreement between shell-model and EDF theory $0 \nu \beta \beta$ decay nuclear matrix elements.

Finally, Fig. 8 shows the angular momentum-parity decomposition of the ${ }^{48} \mathrm{Ca}$ matrix element using $H_{\text {coll }}$, with and without the isoscalar pairing term. When excluded, isoscalar pairing correlations are shown to impact especially the contributions of $J>2$ pairs, which are much more significant for the light-neutrino exchange shown in the left panel. Isoscalar pairing correlations also reduce moderately 
the $J=0^{+}$-pair contribution in both mechanisms, but overall they are not very relevant for heavy neutrinos, as the right panel shows. This minor influence is consistent with the results in Figs. 6 and 7. The contributions of $J>0$ pairs cancel the leading $J=0^{+}$matrix element, dominated by isovector pairing [51]. That the cancellation from $J>0$ pairs is driven by isoscalar pairing supports that these correlations are important to partially recover the $S U(4)$ symmetry of the nuclear states [39], because restoring the symmetry implies a suppression of the matrix elements. The combined insights from Figs. 7 and 8 suggest that $J>0$-pair matrix elements are dominated by the longer-range part of the $0 \nu \beta \beta$ decay operator, which is particularly sensitive to isoscalar pairing correlations.

It is not straightforward how to extend the present analysis beyond $p f$-shell nuclei, because the shell model calculations of $\beta \beta$ emitters besides ${ }^{48} \mathrm{Ca}$ involve configuration spaces which miss spin-orbit partner orbitals, and therefore are not suitable for the use of the collective Hamiltonian in Eq. (9). Therefore the extent to which isoscalar pairing correlations can impact the nuclear matrix elements of ${ }^{76} \mathrm{Ge}$ or ${ }^{136} \mathrm{Xe}$ is uncertain. Nonetheless the estimates of Ref. 39 suggest that isoscalar pairing correlations are also relevant for heavier $\beta \beta$ emitters, although probably less so than in the extreme case, illustrated for ${ }^{48} \mathrm{Ca}$ in the right panel in Fig. 8, where the isoscalar pairing term is completely removed from $H_{\text {coll }}$. On the other hand, it is worth noting that very recently Ref. 81 has suggested, based on neutron-proton transfer reactions, that a shell model interaction similar to the one used to derive the isoscalar pairing strength of $H_{\text {coll }}$ may underestimate the isoscalar pairing strength.

\section{Summary}

Nuclear matrix elements are key to determine the reach of $0 \nu \beta \beta$ decay searches, and to identify the decay channel and obtain information of the underlying new physics once $0 \nu \beta \beta$ decay has been detected. However, two of the preferred many-body methods in nuclear structure, shell model and EDF theory, predict matrix elements that differ by up to a factor three in the standard scenario mediated by light-neutrino exchange. In contrast, a similar comparison for the matrix elements associated with the exchange of heavy neutrinos shows a much milder disagreement of about $50 \%$. This reduced discrepancy is encouraging toward a reliable determination of nuclear matrix elements. In fact the difference between calculations is just about the size of the uncertainty due to short-range correlations.

A comparison of the corresponding matrix elements when correlations in the nuclear states are not permitted shows an even better consistency between the shell model and EDF theory, good to 30\% even for light-neutrino exchange. This suggests that the variance between matrix elements is mainly due to the unequal treatment by the many-body methods of the nuclear structure correlations that are hindered when heavy neutrinos are exchanged. These correspond to the longer-range correlations the $0 \nu \beta \beta$ decay operator is sensitive to. We have used a separable collective Hamiltonian to motivate that the heavy-neutrino-exchange channel is less sensitive to collective correlations. In addition, we have observed that one of the main differences between light- and heavy-neutrino exchanges is due to the role played by isoscalar pairing correlations, which are mainly of long-range character and contribute to cancellations in the matrix elements. Our analysis supports previous works suggesting that isoscalar pairing correlations can be responsible for a sizeable part of the differences between shell-model and EDF theory nuclear matrix elements in the standard scenario of 
exchange of virtual light neutrinos.

Similar studies are needed to understand better the inconsistent matrix elements obtained by various many-body approaches. A more thorough investigation of quadrupole correlations, not very relevant for the nuclei considered in our analysis, is a natural extension of the present work. Other possibilities include a comparison of the matrix elements corresponding to alternative $0 \nu \beta \beta$ decay channels also considering the interacting boson model [18, 82], the QRPA [16, 20] and other novel methods, focusing on the influence of additional collective correlations, or testing the effect of extending the configuration space - e.g. with perturbative [83, 84, variational 85, 86] or improved shell-model [87, 88] techniques - and other aspects of the many-body calculations. Such studies can provide very valuable insights to design strategies to improve matrix element calculations in the near future.

\section{Acknowledgements}

J. M. would like to thank Y. Iwata for many estimulating exchanges in the early stage of this work, V. Cirigliano, E. Mereghetti, T. Otsuka and N. Shimizu for useful discussions, and J. Engel for insightful comments on the manuscript. This work was supported by the Japanese Ministry of Education, Culture, Sports, Science and Technology (MEXT) as Priority Issue on Post-K computer (Elucidation of the Fundamental Laws and Evolution of the Universe) and the Joint Institute for Computational Fundamental Science (JICFuS).

\section{Appendix A. Neutrino potentials}

For completeness we give expressions for the neutrino potentials defined in Sec. 2 used in the calculation of the nuclear matrix elements:

$$
\begin{aligned}
& h_{F}(|\boldsymbol{q}|)=h_{F}^{V V}(|\boldsymbol{q}|), \\
& h_{G T}(|\boldsymbol{q}|)=h_{G T}^{A A}(|\boldsymbol{q}|)+h_{G T}^{A P}(|\boldsymbol{q}|)+h_{G T}^{P P}(|\boldsymbol{q}|)+h_{G T}^{M M}(|\boldsymbol{q}|), \\
& h_{T}(|\boldsymbol{q}|)=h_{T}^{A P}(|\boldsymbol{q}|)+h_{T}^{P P}(|\boldsymbol{q}|)+h_{T}^{M M}(|\boldsymbol{q}|),
\end{aligned}
$$

where the superscripts indicate the particular terms in the hadronic weak one-nucleon current - vector, axial, pseudoscalar or magnetic - generating each contribution. Their explicit form is

$$
\begin{aligned}
& h_{F}^{V V}(|\boldsymbol{q}|)=f_{V}^{2}(|\boldsymbol{q}|), \\
& h_{G T}^{A A}(|\boldsymbol{q}|)=f_{A}^{2}(|\boldsymbol{q}|), \\
& h_{G T}^{A P}(|\boldsymbol{q}|)=-\frac{2}{3} f_{A}^{2}(|\boldsymbol{q}|) \frac{\boldsymbol{q}^{2}}{\left(\boldsymbol{q}^{2}+m_{\pi}^{2}\right)}, \\
& h_{G T}^{P P}(|\boldsymbol{q}|)=\frac{1}{3} f_{A}^{2}(|\boldsymbol{q}|) \frac{\boldsymbol{q}^{4}}{\left(\boldsymbol{q}^{2}+m_{\pi}^{2}\right)^{2}}, \\
& h_{G T}^{M M}(|\boldsymbol{q}|)=\frac{2}{3}\left(\kappa_{p}-\kappa_{n}+1\right)^{2} \frac{g_{V}^{2}}{g_{A}^{2}} f_{V}^{2}(|\boldsymbol{q}|) \frac{\boldsymbol{q}^{2}}{4 M_{N}^{2}}, \\
& h_{T}^{A P}(|\boldsymbol{q}|)=\frac{2}{3} f_{A}^{2}(|\boldsymbol{q}|) \frac{\boldsymbol{q}^{2}}{\left(\boldsymbol{q}^{2}+m_{\pi}^{2}\right)}, \\
& h_{T}^{P P}(|\boldsymbol{q}|)=-\frac{1}{3} f_{A}^{2}(|\boldsymbol{q}|) \frac{\boldsymbol{q}^{4}}{\left(\boldsymbol{q}^{2}+m_{\pi}^{2}\right)^{2}},
\end{aligned}
$$


Neutrinoless $\beta \beta$ decay mediated by light and heavy neutrinos: Role of correlations 18

$$
h_{T}^{M M}(|\boldsymbol{q}|)=\frac{1}{3}\left(\kappa_{p}-\kappa_{n}+1\right)^{2} \frac{g_{V}^{2}}{g_{A}^{2}} f_{V}^{2}(|\boldsymbol{q}|) \frac{\boldsymbol{q}^{2}}{4 M_{N}^{2}},
$$

where $m_{\pi} \approx 138 \mathrm{MeV}$ is the pion mass and $\kappa_{p}-\kappa_{n}+1 \approx 4.70$ [4] is the isovector nucleon magnetic moment. The potentials $h^{A P}(|\boldsymbol{q}|)$ and $h^{P P}(|\boldsymbol{q}|)$ correspond to oneand two-pion-exchange diagrams, respectively. The functions $f_{V}(|\boldsymbol{q}|)$ and $f_{A}(|\boldsymbol{q}|)$ reflect the structure of the nucleon, and are usually parameterized with a dipole form factor:

$$
f_{V}(|\boldsymbol{q}|)=\frac{1}{\left(1+\boldsymbol{q}^{2} / \Lambda_{V}^{2}\right)^{2}}, \quad f_{A}(|\boldsymbol{q}|)=\frac{1}{\left(1+\boldsymbol{q}^{2} / \Lambda_{A}^{2}\right)^{2}},
$$

with $\Lambda_{V}=850 \mathrm{MeV}$ [89] and $\Lambda_{A}=1040 \mathrm{MeV}$ [90 taken from fits to experimental data. At small momentum transfers - not much larger than few times $m_{\pi}$ - equivalent expressions can be derived in the context of chiral EFT [90]:

$$
f_{V}(|\boldsymbol{q}|)=1-2 \frac{\boldsymbol{q}^{2}}{\Lambda_{V}^{2}}, \quad f_{A}(|\boldsymbol{q}|)=1-2 \frac{\boldsymbol{q}^{2}}{\Lambda_{A}^{2}},
$$

with these momentum-transfer corrections only entering at this order in the neutrino potentials not suppressed by $\boldsymbol{q}^{2} / M_{N}^{2}$ 34. In the potentials $h^{A P}$ and $h^{P P}$ involving the pseudoscalar term in the hadronic current, we assumed the Goldberger-Treiman relation for the pion decay constant and pion-nucleon coupling, $f_{\pi} g_{\pi p n} \simeq g_{A} m_{N}$. Corrections to this relation, which at small momentum transfers are also predicted by chiral EFT, are suppressed by $m_{\pi}^{2} / \Lambda_{A}^{2}$ [46, 34. In this work we neglect the additional contribution of loop diagrams and counterterms predicted by chiral effective field theory 91]. First estimates suggest a $\sim 5 \%$ effect for light-neutrino exchange, and a $\sim 10 \%$ impact on heavy-neutrino-exchange nuclear matrix elements for the isotopes considered here.

Table A1 shows the decomposition of the shell model $0 \nu \beta \beta$ decay nuclear matrix elements in Table 1 in terms of the contribution of each neutrino potential, for lightand heavy-neutrino exchange.

\section{References}

[1] Majorana E 1937 Nuovo Cim. 14171

[2] Barabash A S 2015 Nucl. Phys. A 935 52-64

[3] Gando A et al. (KamLAND-Zen Collaboration) 2016 Phys. Rev. Lett. 117082503

[4] Agostini M et al. (GERDA Collaboration) 2017 Nature 54447

[5] Alfonso K et al. (CUORE Collaboration) 2015 Phys. Rev. Lett. 115102502

[6] Gomez-Cadenas J J, Martin-Albo J, Sorel M, Ferrario P, Monrabal F, Munoz-Vidal J, Novella $\mathrm{P}$ and Poves A 2011 JCAP 1106007

[7] Cremonesi O and Pavan M 2014 Adv. High Energy Phys. 2014951432

[8] Dell'Oro S, Marcocci S, Viel M and Vissani F 2016 Adv. High Energy Phys. 20162162659

[9] Engel J and Menéndez J 2017 Rep. Prog. Phys. 80046301

[10] Vergados J, Ejiri H and Šimkovic F 2012 Rept. Prog. Phys. 75106301

[11] Blennow M, Fernandez-Martinez E, Lopez-Pavon J and Menéndez J 2010 JHEP 07096

[12] Gariazzo S, Giunti C, Laveder M, Li Y F and Zavanin E M 2016 J. Phys. G: Nucl. Part. Phys. 43033001

[13] Adhikari R et al. 2017 JCAP $\mathbf{1 7 0 1} 025$

[14] Menéndez J, Poves A, Caurier E and Nowacki F 2009 Nucl. Phys. A 818 139-151

[15] Vaquero N L, Rodríguez T R and Egido J L 2013 Phys. Rev. Lett. 111142501

[16] Šimkovic F, Rodin V, Faessler A and Vogel P 2013 Phys. Rev. C 87045501

[17] Mustonen M T and Engel J 2013 Phys. Rev. C 87064302

[18] Barea J, Kotila J and Iachello F 2015 Phys. Rev. C 91034304

[19] Yao J M, Song L S, Hagino K, Ring P and Meng J 2015 Phys. Rev. C 91024316

[20] Hyvärinen J and Suhonen J 2015 Phys. Rev. C 91024613 
Neutrinoless $\beta \beta$ decay mediated by light and heavy neutrinos: Role of correlations 19

Table A1. Contribution of each neutrino potential to the shell model $0 \nu \beta \beta$ decay nuclear matrix elements for light- and heavy-neutrino exchange, $M^{0 \nu}$ and $M^{0 N}$. Left/right results denote Argonne-/CD-Bonn-type short-range correlations.

\begin{tabular}{ccccccc}
\hline & ${ }^{48} \mathrm{Ca}$ & ${ }^{76} \mathrm{Ge}$ & ${ }^{82} \mathrm{Se}$ & ${ }^{124} \mathrm{Sn}$ & ${ }^{130} \mathrm{Te}$ & ${ }^{136} \mathrm{Xe}$ \\
\hline$M^{0 \nu}$ & $0.93 / 1.02$ & $2.89 / 3.07$ & $2.73 / 2.90$ & $2.76 / 2.96$ & $2.76 / 2.96$ & $2.28 / 2.45$ \\
$-M_{F}^{V V}$ & $0.23 / 0.25$ & $0.54 / 0.59$ & $0.51 / 0.55$ & $0.61 / 0.66$ & $0.62 / 0.67$ & $0.50 / 0.54$ \\
$M_{G T}^{A A}$ & $1.00 / 1.08$ & $2.98 / 3.15$ & $2.81 / 2.97$ & $2.79 / 2.97$ & $2.78 / 2.97$ & $2.31 / 2.45$ \\
$-M_{G T}^{A P}$ & $0.35 / 0.38$ & $0.87 / 0.94$ & $0.82 / 0.89$ & $0.87 / 0.95$ & $0.89 / 0.97$ & $0.73 / 0.79$ \\
$M_{G T}^{P P}$ & $0.12 / 0.13$ & $0.27 / 0.30$ & $0.25 / 0.28$ & $0.27 / 0.30$ & $0.28 / 0.31$ & $0.23 / 0.25$ \\
$M_{G T}^{M M}$ & $0.08 / 0.10$ & $0.18 / 0.22$ & $0.17 / 0.20$ & $0.19 / 0.23$ & $0.19 / 0.23$ & $0.16 / 0.19$ \\
$M_{T}^{A P}$ & $-0.08 /-0.08$ & $-0.01 /-0.01$ & $-0.01 /-0.01$ & $0.01 / 0.01$ & $0.01 / 0.01$ & $0.01 / 0.01$ \\
$M_{T}^{P P}$ & $0.03 / 0.03$ & $0.00 / 0.00$ & $0.00 / 0.00$ & $-0.01 /-0.01 /$ & $-0.01 /-0.01$ & $-0.01 /-0.01$ \\
$M_{T}^{M M}$ & $-0.01 /-0.01$ & $0.00 / 0.00$ & $0.00 / 0.00$ & $0.00 / 0.00$ & $0.00 / 0.00$ & $0.00 / 0.00$ \\
\hline$M^{0 N}$ & $48 / 73$ & $130 / 188$ & $121 / 175$ & $141 / 203$ & $146 / 210$ & $116 / 167$ \\
$-M_{F}^{V V}$ & $21 / 26$ & $49 / 59$ & $46 / 55$ & $52 / 63$ & $54 / 65$ & $43 / 52$ \\
$M_{G T}^{A A}$ & $65 / 85$ & $150 / 196$ & $140 / 183$ & $158 / 208$ & $163 / 214$ & $130 / 171$ \\
$-M_{G T}^{A P}$ & $29 / 42$ & $63 / 91$ & $59 / 84$ & $68 / 98$ & $70 / 101$ & $56 / 80$ \\
$M_{G T}^{P P}$ & $10 / 16$ & $21 / 33$ & $19 / 31$ & $22 / 36$ & $23 / 37$ & $18 / 30$ \\
$M_{G T}^{M M}$ & $-2 / 7$ & $-6 / 14$ & $-6 / 13$ & $-5 / 17$ & $-5 / 17$ & $-4 / 14$ \\
$M_{T}^{A P}$ & $-12 /-12$ & $-2 /-2$ & $-2 /-2$ & $2 / 2$ & $3 / 3$ & $2 / 2$ \\
$M_{T}^{P P}$ & $5 / 5$ & $1 / 1$ & $1 / 1$ & $-1 /-1$ & $-1 /-1$ & $-1 /-1$ \\
$M_{T}^{M M}$ & $-2 /-2$ & $0 / 0$ & $0 / 0$ & $0 / 0$ & $0 / 0$ & $0 / 0$ \\
\hline
\end{tabular}

[21] Horoi M and Neacsu A 2016 Phys. Rev. C 93024308

[22] Iwata Y, Shimizu N, Otsuka T, Utsuno Y, Menéndez J, Honma M and Abe T 2016 Phys. Rev. Lett. 116112502

[23] Yao J M and Engel J 2016 Phys. Rev. C 94014306

[24] Pastore S, Baroni A, Carlson J, Gandolfi S, Pieper S C, Schiavilla R and Wiringa R B 2017 (Preprint arXiv:1709.03592)

[25] Shanahan P E, Tiburzi B C, Wagman M L, Winter F, Chang E, Davoudi Z, Detmold W, Orginos K and Savage M J 2017 Phys. Rev. Lett. 119062003

[26] Tiburzi B C, Wagman M L, Winter F, Chang E, Davoudi Z, Detmold W, Orginos K, Savage M J and Shanahan P E 2017 Phys. Rev. D 96054505

[27] Benes P, Faessler A, Simkovic F and Kovalenko S 2005 Phys. Rev. D 71077901

[28] Rath P K, Chandra R, Chaturvedi K, Lohani P, Raina P K and Hirsch J G 2013 Phys. Rev. C 88064322

[29] Faessler A, González M, Kovalenko S and Šimkovic F 2014 Phys. Rev. D 90096010

[30] Neacsu A and Horoi M 2015 Phys. Rev. C 91024309

[31] Barea J, Kotila J and Iachello F 2015 Phys. Rev. D 92093001

[32] Song L S, Yao J M, Ring P and Meng J 2017 Phys. Rev. C 95024305

[33] Pastore S, Carlson J, Cirigliano V, Dekens W, Mereghetti E and Wiringa R B 2017 (Preprint arXiv: 1710.05026

[34] Menéndez J, Gazit D and Schwenk A 2011 Phys. Rev. Lett. 107062501

[35] Menéndez J (Preprint 1605.05059)

[36] Rath P K, Chandra R, Raina P K, Chaturvedi K and Hirsch J G 2012 Phys. Rev. C 85014308

[37] Menéndez J, Rodríguez T R, Martínez-Pinedo G and Poves A 2014 Phys. Rev. C 90024311

[38] Dufour M and Zuker A P 1996 Phys. Rev. C 54 1641-1660 
Neutrinoless $\beta \beta$ decay mediated by light and heavy neutrinos: Role of correlations 20

[39] Menéndez J, Hinohara N, Engel J, Martínez-Pinedo G and Rodríguez T R 2016 Phys. Rev. C 93014305

[40] Kotila J and Iachello F 2012 Phys. Rev. C 85034316

[41] Olive K A et al. (Particle Data Group) 2014 Chin. Phys. C 38090001

[42] Šimkovic F, Pantis G, Vergados J D and Faessler A 1999 Phys. Rev. C 60055502

[43] Muto K 1994 Nucl. Phys. A $\mathbf{5 7 7}$ 415C-420C

[44] Sen'kov R A and Horoi M 2013 Phys. Rev. C 88064312

[45] Sen'kov R A and Horoi M 2014 Phys. Rev. C 90051301

[46] Šimkovic F, Faessler A, Müther H, Rodin V and Stauf M 2009 Phys. Rev. C 79055501

[47] Prézeau G, Ramsey-Musolf M and Vogel P 2003 Phys. Rev. D 68034016

[48] Cirigliano V, Dekens W, Graesser M and Mereghetti E 2017 Phys. Lett. B 769 460-464

[49] Nicholson A, Berkowitz E, Chang C C, Clark M A, Joo B, Kurth T, Rinaldi E, Tiburzi B, Vranas P and Walker-Loud A (Preprint arXiv:1608.04793)

[50] Cirigliano V, Dekens W, de Vries J, Graesser M L and Mereghetti E 2017 (Preprint arXiv: 1708.09390

[51] Caurier E, Menéndez J, Nowacki F and Poves A 2008 Phys. Rev. Lett. 100052503

[52] Poves A, Sánchez-Solano J, Caurier E and Nowacki F 2001 Nucl. Phys. A 694 157-198

[53] Hjorth-Jensen M, Kuo T and Osnes E 1995 Phys. Rep. 261125

[54] Gniady A, Caurier E and Nowacki F Unpublished

[55] Sieja K, Martinez-Pinedo G, Coquard L and Pietralla N 2009 Phys. Rev. C 80054311

[56] Caurier E, Nowacki F, Poves A and Sieja K 2010 Phys. Rev. C 82064304

[57] Klos P, Menéndez J, Gazit D and Schwenk A 2013 Phys. Rev. D 88083516 [Erratum: Phys. Rev. D 89, 029901 (2014)]

[58] Vietze L, Klos P, Menéndez J, Haxton W C and Schwenk A 2015 Phys. Rev. D 91043520

[59] Caurier E, Martínez-Pinedo G, Nowacki F, Poves A and Zuker A P 2005 Rev. Mod. Phys. 77 427

[60] Šimkovic F, Faessler A, Rodin V, Vogel P and Engel J 2008 Phys. Rev. C 77045503

[61] Faessler A, Fogli G L, Lisi E, Rotunno A and Šimkovic F 2011 Phys. Rev. D 83113015

[62] Rodríguez T R and Martínez-Pinedo G 2013 Phys. Lett. B 719174

[63] Shimizu N, Menéndez J and Yako K 2017 (Preprint arXiv:1709.01088)

[64] Lisi E, Rotunno A and Šimkovic F 2015 Phys. Rev. D 92093004

[65] Deppisch F and Päs H 2007 Phys. Rev. Lett. 98232501

[66] Gehman V M and Elliott S R 2007 J. Phys. G: Nucl. Part. Phys. 34667

[67] Horoi M and Neacsu A 2016 Phys. Rev. D 93113014

[68] Rodríguez T R and Martínez-Pinedo G 2011 Prog. Part. Nucl. Phys. 66 436-440

[69] Rodríguez T R, Poves A and Nowacki F 2016 Phys. Rev. C 93054316

[70] Rodríguez T 2017 J. Phys. G: Nucl. Part. Phys. 44034002

[71] Caurier E, Nowacki F and Poves A 2008 Eur. Phys. J. A 36 195-200

[72] Vogel P and Zirnbauer M R 1986 Phys. Rev. Lett. 57 3148-3151

[73] Engel J, Vogel P and Zirnbauer M R 1988 Phys. Rev. C 37731

[74] Hinohara N and Engel J 2014 Phys. Rev. C 90031301

[75] Rodríguez T R and Martínez-Pinedo G 2010 Phys. Rev. Lett. 105252503

[76] Menéndez J, Poves A, Caurier E and Nowacki F 2011 J. Phys. Conf. Ser. 267012058

[77] Fang D L, Faessler A, Rodin V and Šimkovic F 2011 Phys. Rev. C 83034320

[78] Song L S, Yao J M, Ring P and Meng J 2014 Phys. Rev. C 90054309

[79] Kidd M F, Esterline J H, Finch S W and Tornow W 2014 Phys. Rev. C 90055501

[80] Engel J and Vogel P 2004 Phys. Rev. C 69034304

[81] Ayyad Y et al. 2017 Phys. Rev. C 96021303

[82] Van Isacker P, Engel J and Nomura K 2017 Phys. Rev. C 96064305

[83] Holt J D and Engel J 2013 Phys. Rev. C 87064315

[84] Tsunoda N, Otsuka T, Shimizu N, Hjorth-Jensen M, Takayanagi K and Suzuki T 2017 Phys. Rev. C 95021304

[85] Jiao C F, Engel J and Holt J D 2017 Phys. Rev. C 96054310

[86] Jiao C F, Horoi M and Neacsu A 2017 (Preprint arXiv:1709.05313)

[87] Shimizu N, Abe T, Honma M, Otsuka T, Togashi T, Tsunoda Y, Utsuno Y and Yoshida T 2017 Phys. Scr. 92063001

[88] Togashi T, Tsunoda Y, Otsuka T and Shimizu N 2016 Phys. Rev. Lett. 117172502

[89] Dumbrajs O, Koch R, Pilkuhn H, Oades G C, Behrens H, De Swart J J and Kroll P 1983 Nucl. Phys. B 216 277-335

[90] Bernard V, Elouadrhiri L and Meissner U G 2002 J. Phys. G: Nucl. Part. Phys. 28 R1-R35

[91] Cirigliano V, Dekens W, Mereghetti E and Walker-Loud A 2017 (Preprint arXiv:1710.01729) 